\title{
Self-Assembling Protein-Polymer Bioconjugates for Surfaces with Antifouling Features and Low Nonspecific Binding
}

\author{
Yingying Liu, ${ }^{\dagger}$ Tarja K. Nevanen, ${ }^{\dagger}$ Arja Paananen, ${ }^{\dagger}$ Kristian Kempe, $^{\ddagger, \S \odot \text { Paul Wilson, }}{ }^{\ddagger \oplus}$ \\ Leena-Sisko Johansson, ${ }^{\perp}$ Jussi J. Joensuu, ${ }^{\dagger}$ Markus B. Linder, ${ }^{\perp}{ }^{\circ}$ David M. Haddleton, ${ }^{+\infty}$ \\ and Roberto Milani* ${ }^{*} \dagger$ \\ ${ }^{\dagger}$ VTT Technical Research Centre of Finland Ltd., P.O. Box 1000, FI-02044 Espoo, Finland \\ "Department of Chemistry, University of Warwick, CV4 7AL Coventry, United Kingdom \\ ${ }^{\S}$ ARC Centre of Excellence in Convergent Bio-Nano Science and Technology, Monash Institute of Pharmaceutical Sciences, \\ Monash University, VIC 3052, Parkville, Australia \\ ${ }^{\perp}$ Department of Bioproducts and Biosystems, Aalto University, P.O. Box 11000, FI-00076 Aalto, Finland
}

\section{Supporting Information}

ABSTRACT: A new method is demonstrated for preparing antifouling and low nonspecific adsorption surfaces on poorly reactive hydrophobic substrates, without the need for energyintensive or environmentally aggressive pretreatments. The surface-active protein hydrophobin was covalently modified with a controlled radical polymerization initiator and allowed to self-assemble as a monolayer on hydrophobic surfaces, followed by the preparation of antifouling surfaces by $\mathrm{Cu}(0)$ mediated living radical polymerization of poly(ethylene glycol) methyl ether acrylate (PEGA) performed in situ. By taking advantage of hydrophobins to achieve at the same time the immobilization of protein $\mathrm{A}$, this approach allowed to prepare surfaces for IgG1 binding featuring greatly reduced

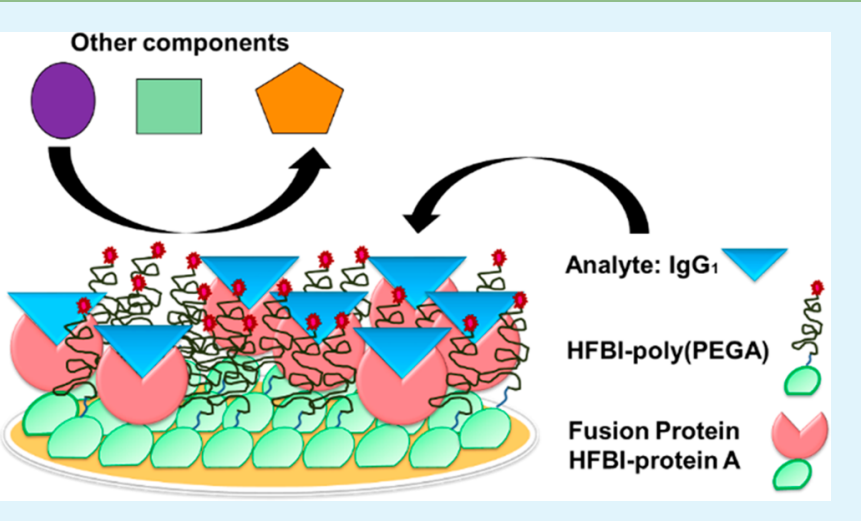
nonspecific adsorption. The success of the surface modifica-

tion strategy was investigated by contact angle, XPS, and AFM characterization, while the antifouling performance and the reduction of nonspecific binding were confirmed by QCM-D measurements.

KEYWORDS: antifouling surfaces, nonspecific binding, biosensors, hydrophobin, surface-initiated living radical polymerization

\section{INTRODUCTION}

Surfaces with antifouling properties find major application in the biomedical field, in particular where the reduction of nonspecific protein adsorption and cell adhesion is a necessary requirement, e.g., for prostheses and implantable devices to avoid effects such as inflammation and fibrosis. ${ }^{1-5}$ The minimization of nonspecific binding is important, however, also in biosensing applications since nonspecific adsorption may produce a false response, thus decreasing the detection specificity and sensitivity of a device. ${ }^{6-8}$

However, the introduction of antifouling features through surface modification can be a significant challenge in the case of poorly reactive materials, like some of the most commonly used hydrophobic polymers (e.g., polyolefins, polystyrene, poly(dimethylsiloxane), or Teflon). Activation treatments are typically required which are energy intensive or environmentally aggressive, such as UV-irradiation, plasma, or strongly oxidizing chemicals. One simple, fast, and environmentally friendly means of achieving the modification of chemically inert surfaces is offered by hydrophobins, which are natural amphiphilic proteins produced by filamentous fungi. ${ }^{9-11}$
Thanks to their unique Janus-like structure, which features a hydrophobic patch composed by amino acids with hydrophobic side chains, these proteins can self-assemble spontaneously on hydrophobic surfaces from aqueous solutions and form robust monolayers which expose a reactive hydrophilic side. Previous work showed that they can be used as surface modification agents for poorly reactive surfaces such as graphite, ${ }^{12}$ diamond-like carbon, ${ }^{13}$ Teflon, ${ }^{14-16}$ poly(vinylidene fluoride), ${ }^{17}$ polystyrene, and polyethylene. ${ }^{18}$ In particular, hydrophobins were shown to permit the successful immobilization of enzymes, antibodies, and other proteins as sensing modules in biosensor devices, either by electrostatic interactions or by expression as fusion proteins. ${ }^{19-25}$

Among the materials possessing antifouling properties, poly(ethylene glycol) (PEG) and its derivatives are wellknown and have been studied and successfully employed over decades of research. ${ }^{1,5,26-29}$ In recent years it was shown that a

Received: November 13, 2018

Accepted: December 19, 2018

Published: December 19, 2018 

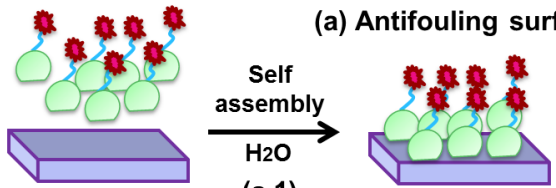

(a.1)

(b) Low non-specific binding surface
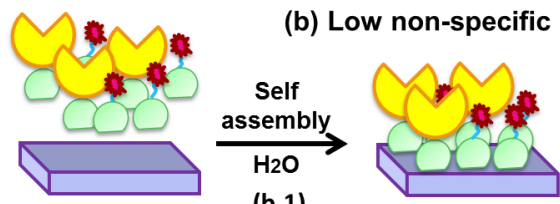

(b.1)

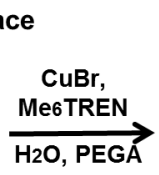

(a.2)

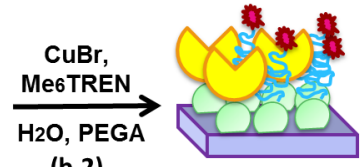

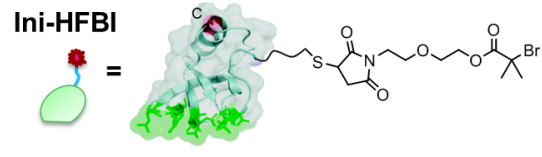

Poly(PEGA)-HFBI

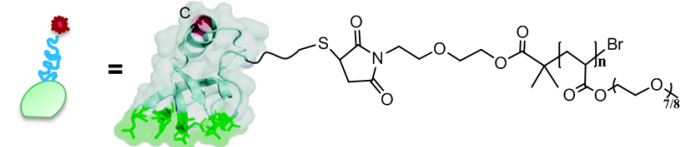

HFBI-ProtA

= Fusion protein HFBI-protein A

Figure 1. Surface-initiated polymerization of poly(PEGA). (a) Process of preparation of an antifouling surface: (a.1) self-assembly of Ini-HFBI on a hydrophobic surface; (a.2) polymerization of PEGA from Ini-HFBI. (b) Process of preparation of a low nonspecific binding surface: (b.1) coassembly of Ini-HFBI and fusion protein HFBI-ProtA on a hydrophobic surface; (b.2) polymerization of PEGA from the mixed Ini-HFBI/HFBIProtA layer.

range of hydrophilic PEG-based comb/brush polymers can be prepared fast and efficiently in mild conditions, including aqueous environment, by $\mathrm{Cu}(0)$-mediated living radical polymerization. ${ }^{30-37}$ For example, poly(methacrylates) were grown successfully on various surfaces to prevent nonspecific protein adsorption, ${ }^{32,38}$ and graphene oxide (GO) was functionalized with poly(ethylene glycol) ethyl ether methacrylate (PEGEEMA) to improve GO dispersibility in various solvents and exhibit thermoresponsive wettability. ${ }^{31}$

In this study we report the preparation and use of a macroinitiator obtained by site-specific covalent bioconjugation of a living radical polymerization initiator to the hydrophobin NCysHFBI. This new macroinitiator Ini-HFBI was first allowed to self-assemble as a film on hydrophobic surfaces by simple substrate immersion in aqueous solution. Subsequently, $\mathrm{Cu}(0)$-mediated living radical polymerization under mild conditions of poly(ethylene glycol)methyl ether acrylate (PEGA) was used to prepare in situ an antifouling layer of hydrophobin/poly(PEGA) bioconjugate via the "grafting from" strategy (Figure 1). The same method was then applied to obtain a surface for IgG1 binding with low nonspecific adsorption, prepared as a mixed layer of the same hydrophobin/poly(PEGA) bioconjugate together with an engineered fusion of the hydrophobin HFBI with protein $A$ (HFBI-ProtA). The composition and morphology of the surfaces were investigated by spectroscopic and microscopic techniques, and their antifouling performance and protein binding features were studied by quartz crystal microbalance with dissipation monitoring (QCM-D). The reduction of nonspecific binding was assessed by investigating the selective adsorption of IgG1 in the presence of a large excess of BSA, which is often used as blocker for nonspecific binding sites, for example, in immunohistochemistry.

\section{MATERIALS AND METHODS}

Reagents. The P-type single-side polished silicon wafer (100) used as the substrate was obtained from Siegert wafer $\mathrm{GmbH}$, Germany. The gold-coated quartz crystal sensors (QSX 301) were purchased from Biolin Scientific. Poly(ethylene glycol)methyl ether acrylate (PEGA; $\left.M_{\mathrm{n}}=480 \mathrm{~g} \mathrm{~mol}^{-1}\right)$, copper $(\mathrm{I})$ bromide $(\mathrm{CuBr})$, tris[2-(dimethylamino)ethyl] amine (Me6-TREN), trifluoroacetic acid (TFA), 1-hexanethiol, chlorotrimethylsilane, decane, acetonitrile $(\mathrm{ACN})$, ethanol $(\mathrm{EtOH})$, and acetone were purchased from SigmaAldrich. All reagents were used as received. NCysHFBI was produced using recombinant strains of $T$. reesei, purified by RP-HPLC as described previously ${ }^{39}$ and lyophilized before use. Bovine serum albumin (BSA), immunoglobulin Lambda from human myeloma plasma (IgG1), and anti-IgG( $\gamma$-chain specific)-gold $5 \mathrm{~nm}$ colloidal gold were purchased from Sigma-Aldrich. The HFBI-ProteinA fusion protein produced in transient expression mode in Nicotiana tabacum plants via agroinfiltration ${ }^{40}$ was produced and purified as previously described. ${ }^{23}$

Preparation of the Ini-HFBI Macroinitiator. The maleimidic initiator 2-bromoisobutyrate ethoxyethyl maleimide was synthesized according to the literature. ${ }^{41,42}$ NCysHFBI was first reduced as previously described ${ }^{43}$ to reverse the dimerization which occurs by formation of disulfide bridges between the exposed cysteines situated close to the $\mathrm{N}$ terminus of the proteins. The same cysteine residues were then used for site-specific conjugation of the maleimide initiator to the protein. In a typical synthesis, reduced NCysHFBI $(4.7 \mathrm{mg})$ was dissolved in a mixture of acetonitrile $(0.55 \mathrm{~mL})$ and phosphate buffer $(2.45 \mathrm{~mL}, 0.1 \mathrm{M}, \mathrm{pH} 7.2)$. A solution of 2-bromoisobutyrate ethoxyethyl maleimide $(3.3 \mathrm{mg})$ in ACN $(0.5 \mathrm{~mL})$ was added. The vial containing the mixture was briefly flushed with nitrogen, wrapped in aluminum foil, and gently stirred at room temperature for $4 \mathrm{~h}$. The mixture was then centrifuged at $4000 \mathrm{rpm}$ and $+20^{\circ} \mathrm{C}$ for $10 \mathrm{~min}$, followed by purification by reverse phase HPLC on a Resource RPC column using a gradient of water/ACN (both containing $0.1 \%$ TFA). Ini-HFBI was subsequently freeze-dried and recovered typically in $65-70 \%$ yield. MALDI-TOF: $m / z=9012.7$.

MALDI-TOF Spectrometry. An Autoflex II instrument (Bruker Daltonics Bremen, Germany) equipped with a UV/N2 laser (337 $\mathrm{nm} / 100 \mu \mathrm{J}$ ) was used to perform mass spectrometric analyses. Saturated $\alpha$-cyano-4-hydroxycinnamic acid (Sigma-Aldrich) in a $1: 2$ $(\mathrm{v} / \mathrm{v})$ mixture of $\mathrm{ACN}$ and aqueous $0.1 \%(\mathrm{v} / \mathrm{v})$ TFA was used as a matrix. The purified proteins were dissolved in the same solvent mixture at about $1 \mathrm{mg} \mathrm{mL}{ }^{-1}$ concentration, mixed with the matrix solutions in $1: 1(\mathrm{v} / \mathrm{v})$ ratio, and applied on a stainless steel target plate in $0.5 \mu \mathrm{L}$ aliquots. The sample spot was dried in air at room temperature. The mass spectrum $(4-20 \mathrm{kDa})$ was measured in linear positive-ion mode, and protein standard solution I (Bruker Daltonics) was used for the external molecular mass calibration.

Interfacial Tension Measurements. Interface tensiometric measurements were performed on a CAM 200 (KSV Instruments Ltd.), and the built-in software OneAttension was used for data processing. A $15 \mu \mathrm{L}$ droplet of HFBI or Ini-HFBI aqueous solution at various concentrations (heavy phase) was left hanging in decane (light phase) within a quartz cuvette from the syringe needle. The selfassembly ability of Ini-HFBI at different $\mathrm{pH}$ was studied as well by using the following buffers at $10 \mathrm{mM}$ concentration: sodium acetateacetic acid ( $\mathrm{pH} 4$ and 5), sodium phosphate ( $\mathrm{pH} 6$ and 7), Tris- $\mathrm{HCl}$ $(\mathrm{pH} 8)$, and glycine- $\mathrm{NaOH}(\mathrm{pH} \mathrm{9.0)}$. The interfacial energies were obtained from the fitting of the droplet shape by the Young-Laplace method. 
Preparation of Poly(PEGA)-HFBI Layers. Silicon wafer slides (1 $\times 1 \mathrm{~cm}^{2}$ size) were cleaned and silanized with chlorotrimethysilane to obtain a model hydrophobic surface following the procedure reported in the Supporting Information. A silanized silicon slide was immersed in Ini-HFBI $\left(50 \mu \mathrm{g} \mathrm{mL}^{-1}\right)$ aqueous solution for $45 \mathrm{~min}$. At the same time, $\mathrm{H}_{2} \mathrm{O}$ (HPLC grade, $1 \mathrm{~mL}$ ) and Me6-TREN (5.35 $\mu \mathrm{L}, 0.02$ $\mathrm{mmol}$ ) were added to a $20 \mathrm{~mL}$ glass vial fitted with a magnetic stir bar and a rubber septum. The mixture was deoxygenated in a $\mathrm{N}_{2}$ stream for $10 \mathrm{~min}$. $\mathrm{CuBr}(2.87 \mathrm{mg}, 0.02 \mathrm{mmol})$ was then carefully added while keeping $\mathrm{N}_{2}$ flowing. The solution was stirred for $15 \mathrm{~min}$ to generate a bluish-green solution of $\mathrm{CuBr}_{2} / \mathrm{Me} 6-\mathrm{TREN}$ and a suspension of $\mathrm{Cu}(0)$ powder. Then stirring was stopped and one Ini-HFBI covered silicon wafer slide was carefully immersed into the solution, which was again bubbled with $\mathrm{N}_{2}$ for at least $30 \mathrm{~min}$. At the same time, a mixture of $\mathrm{H}_{2} \mathrm{O}$ (HPLC grade, $2 \mathrm{~mL}$ ) and PEGA (960 $\mathrm{mg}, 881 \mu \mathrm{L}, 2 \mathrm{mmol}$ ) was charged into a glass vial equipped with a rubber septum, and the mixture was deoxygenated for at least $30 \mathrm{~min}$. After that, the deoxygenated monomer solution was transferred via a well-deoxygenated syringe through the septum to the bottom of the glass vial with $\mathrm{Cu}(0) / \mathrm{CuBr}_{2} / \mathrm{Me} 6-\mathrm{TREN} /$ silicon wafer slide. Polymerization was performed at room temperature under a nitrogen atmosphere overnight (for the mixed Ini-HFBI/HFBI-ProtA layers, the polymerization time was $2 \mathrm{~h}$ ), and then the polymer-grafted silicon wafer slide was taken out of the solution, rinsed with abundant Milli- $Q$ water, and dried under nitrogen flow. The same procedure was applied to a silanized $\mathrm{Si}$ wafer previously half-covered with Teflon tape for the AFM study.

Water Contact Angle (WCA) and Surface Free Energy. The contact angles of all surfaces were measured using a CAM 200 instrument (KSV) with $5 \mu \mathrm{L}$ droplets for Milli-Q water, diiodomethane, and benzyl alcohol using the sessile drop method. The drop profiles were fitted by using the Young-Laplace method. Contact angles were determined as an average from three parallel experiments on different areas of the samples by the OneAttension software. The surface energy was calculated from the contact angle values with water, diiodomethane, and benzyl alcohol using the Owens-Wendt method. ${ }^{44}$ All samples were rinsed with Milli-Q water and dried under $\mathrm{N}_{2}$ flow before measurement.

X-ray Photoelectron Spectroscopy (XPS). Surface chemistry was assessed by XPS (AXIS Ultra by Kratos). For the analyses, samples were mounted on a sample holder with UHV compatible carbon tape, together with an in situ reference sample of $100 \%$ ashfree cellulose filter paper, ${ }^{45}$ and pre-evacuated overnight. All experiments were performed using monochromatic $\mathrm{Al} \mathrm{K}$ irradiation at low irradiation $(100 \mathrm{~W})$ and under neutralization. Low resolution wide energy range spectra were recorded together with high resolution scans of $\mathrm{C} 1 \mathrm{~s}, \mathrm{O} 1 \mathrm{~s}, \mathrm{~N} 1 \mathrm{~s}$, and $\mathrm{Si} 2 \mathrm{p}$ regions on at least three locations for each sample. With the parameters used, analysis area is less than $1 \mathrm{~mm}^{2}$, while the analysis depth of XPS is much smaller, $<10 \mathrm{~nm}$. The data were analyzed using CasaXPS. Wide scans were used in surface elemental analysis, C 1s high resolution data were curve fitted with Gaussian components for a more detailed chemical analysis, ${ }^{46}$ and the binding energies of all spectra were adjusted with the help of the in situ reference and the CC component present in all samples (i.e., carbon atoms without oxygen neighbors).

Atomic Force Microscopy (AFM). Topography images were captured with NanoScopeV Multimode8 AFM (E scanner, Bruker). All images were recorded using cantilevers with a tip radius of $8 \mathrm{nM}$ (MikroMasch, $f=325 \mathrm{kHz}, k=40 \mathrm{~N} \mathrm{~m}^{-1}$ ) in tapping mode in air with scan rate $1.4 \mathrm{~Hz}$. The Nanoscope Analysis software (Bruker) was used for image processing and analysis. The images were only flattened to remove possible tilt in the image data, with no further processing.

Scanning Electron Microscopy (SEM). Morphological features were imaged with a Zeiss Sigma VP scanning electron microscope. The samples were attached on aluminum SEM stubs with carbon tape and coated with palladium/platinum by using a Leica EM ACE600 high vacuum sputter coater ( $35 \mathrm{~mA}, 4 \mathrm{~nm}$ thickness) prior to imaging. All images were recorded in Secondary Electron (SE) mode with inlens detector under an acceleration voltage of $2.0 \mathrm{kV}$. The labeling with anti-IgG( $\gamma$-chain specific)-gold was performed by diluting 10 fold the solution received from the supplier with $20 \mathrm{mM}$ Tris buffer ( $\mathrm{pH} 8$ ) containing $0.05 \mathrm{M} \mathrm{NaCl}$ and $0.05 \%$ TWEEN, followed by sample incubation for $1 \mathrm{~h}$ and rinsing with Milli-Q.

Antifouling Features of Poly(PEGA)-HFBI Layers. Quartz crystal microbalance with dissipation monitoring (QCM-D; Q-Sense E4 system, Biolin Scientific, Sweden) was first used to measure the adsorption of BSA on hydrophobin surfaces with and without the poly(PEGA) layer, using the wild-type parent protein HFBI as a control layer. For all QCM-D experiments reported in this work, the surface-adsorbed masses were estimated with the QTools software using the viscoelastic Voigt model.

The QCM gold sensor disks were first coated with 1-hexanethiol to obtain a model hydrophobic surface. The gold sensor disks were first cleaned in a UV/ozone chamber for $10 \mathrm{~min}$ and immersed in a heated $\left(75{ }^{\circ} \mathrm{C}\right) \mathrm{H}_{2} \mathrm{O} / \mathrm{NH}_{3} / \mathrm{H}_{2} \mathrm{O}_{2}$ mixture $(5: 1: 1)$ for another $10 \mathrm{~min}$ followed by thorough rinsing with Milli-Q water and drying with $\mathrm{N}_{2}$. Then dried sensor disks were treated in the UV/ozone chamber for another $10 \mathrm{~min}$ before immersing in pure $\mathrm{EtOH}$ for $2 \mathrm{~min}$ to sensitize the surfaces. The wet sensor disks were then immersed in 1hexanethiol/EtOH $(0.05 \mathrm{M})$ solution overnight at room temperature. Finally, they were rinsed with pure EtOH and Milli-Q water and dried with $\mathrm{N}_{2}$. Then the hydrophobic gold sensor disks were immersed in aqueous solutions of Ini-HFBI $\left(50 \mu \mathrm{g} \mathrm{mL}^{-1}\right)$ and HFBI $\left(50 \mu \mathrm{g} \mathrm{mL}^{-1}\right)$ for $50 \mathrm{~min}$ and rinsed with water to remove excess hydrophobin. The sensor modified with HFBI was dried with $\mathrm{N}_{2}$ after deposition and conserved for QCM-D as a control sample. After rinsing with Milli- $Q$ water, the sensor modified with Ini-HFBI was immediately transferred into the solution containing the reagents for the polymerization of PEGA, which was performed as described above. After polymerization, the sensor disk was rinsed with water and dried with $\mathrm{N}_{2}$ prior to mounting into the measurement chamber of the QCM-D.

For the antifouling tests, BSA was first dissolved in sodium acetate-acetic acid buffer $(10 \mathrm{mM})$ at $\mathrm{pH} 5.5$ to a concentration of 1 $\mathrm{mg} \mathrm{mL} \mathrm{m}^{-1}$. During the QCM-D measurements, the BSA solution was pumped through the measurement chambers at a $0.1 \mathrm{~mL} \mathrm{~min}^{-1}$ flow rate for $6 \mathrm{~min}$. The flow was then interrupted, and the system was allowed to stabilize for $110 \mathrm{~min}$; then rinsing was performed with the buffer solution until the signal stabilized.

Surfaces for IgG1 Binding with Reduced Nonspecific Adsorption. The coassembly of the fusion protein HFBI-ProtA with the wild-type hydrophobin HFBI was first investigated by QCMD using hexanethiol-coated gold sensor disks to study the IgG1binding capacity of different mixed layers. During the QCM-D measurement, mixtures of HFBI-ProtA and wild-type HFBI in different HFBI-ProtA/HFBI molar ratios (0/100, 20/80, 40/60, and $60 / 40)$, for a total concentration of $2.27 \mu \mathrm{M}$ in sodium phosphate $(10 \mathrm{mM})$ at $\mathrm{pH} 7$ were pumped into the chambers at $0.1 \mathrm{~mL} \mathrm{~min}^{-1}$ flow for $6 \mathrm{~min}$. The sensors were then incubated for ca. $60 \mathrm{~min}$ in zero-flow conditions. After rinsing with buffer solution until a stable baseline was obtained again, IgG1 solutions of increasing concentration $(8,80$, and $800 \mathrm{nM})$ were injected in sequence into the chambers at a flow rate of $0.1 \mathrm{~mL} \mathrm{~min}{ }^{-1}$ for $6 \mathrm{~min}^{23}$ The sensors were allowed to equilibrate in zero-flow condition for ca. $70 \mathrm{~min}$ and rinsed with the running buffer in between injections to remove excess IgG1.

To verify the reduction of nonspecific protein adsorption on IgG1 binding surfaces, a mixed layer of Ini-HFBI and HFBI-ProtA (40\% molar ProtA in solution) was first assembled outside the QCM-D instrument on a hexanethiol-coated QCM sensor using a total protein concentration of $100 \mu \mathrm{g} \mathrm{mL}^{-1}$ and then performing the polymerization of PEGA using the same procedures described above for pure Ini-HFBI layers. These surface-modified sensors were then used in QCM-D protein adsorption experiments performed under the same conditions described above for the antifouling tests $\left(0.1 \mathrm{~mL} \mathrm{~min}^{-1}\right.$ flow, $700 \mu \mathrm{L}$ volume of protein solution), using solutions of BSA (1 $\mathrm{mg} \mathrm{mL} L^{-1}, 15 \mu \mathrm{M}$ ) and BSA/IgG1 mixtures (BSA: $15 \mu \mathrm{M}$; IgG1: 8 or $80 \mathrm{nM})$ in sodium acetate-acetic acid buffer $(10 \mathrm{mM})$ at $\mathrm{pH} 5.5$. 


\section{RESULTS AND DISCUSSION}

Characterization of the Macroinitiator Ini-HFBI. To prepare the macroinitiator Ini-HFBI, 2-bromoisobutyrate ethoxyethyl maleimide was covalently bound by site-specific conjugation to NCysHFBI, a genetically engineered variant of the wild-type hydrophobin HFBI. ${ }^{39}$ NCysHFBI features an added strand of 13 amino acids to the N-terminus of HFBI, which contains one cysteine amino acid that can be selectively reacted with compounds containing a maleimide functional group. MALDI-TOF mass spectrometry confirmed the success of the derivatization, as the modified protein displayed a molecular weight of $9012 \mathrm{Da}$ (Figure S1).

The ability of the modified hydrophobin Ini-HFBI to assemble at interfaces and reduce interfacial tension was investigated for protein aqueous solutions with different concentrations at the interface between water and decane, and the results were compared to those obtained with the wildtype protein HFBI (Figure 2, green curve). Droplet

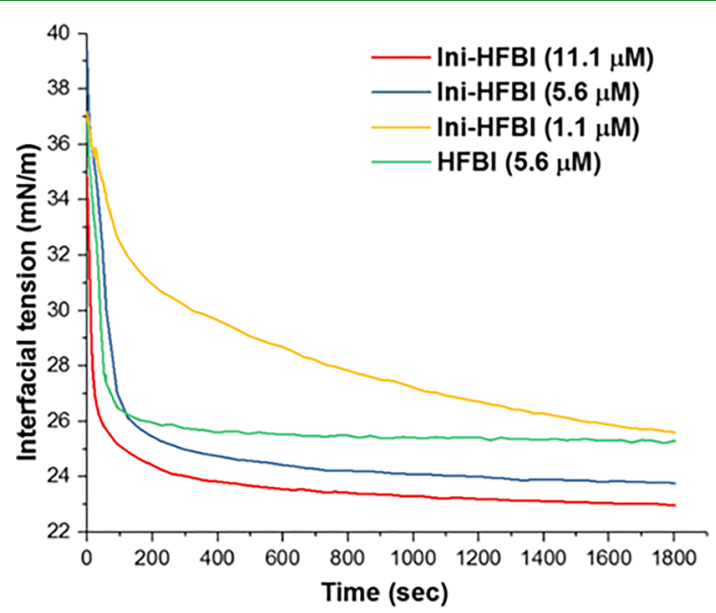

Figure 2. Plots of interfacial tension versus time at the interface between aqueous protein solutions and decane.

tensiometry shows that the typical surface activity of hydrophobins is retained in Ini-HFBI. The surface tension decreased rapidly within the first $100 \mathrm{~s}$, which is attributed to protein adsorption at the interface. This was followed by a subsequent slower reduction of interface tension due to a rearrangement of the protein film, until a stable state was reached. This behavior was largely independent of the $\mathrm{pH}$ as no significant changes or clear trends could be observed when varying the $\mathrm{pH}$ between 4 and 9 (Figure S2).

To determine the most suitable conditions for protein film assembly, the kinetics of interface energy reduction of IniHFBI was studied at 100,50 , and $10 \mu \mathrm{g} / \mathrm{mL}(11.1,5.6$, and 1.1 $\mu \mathrm{M}$ ) concentration (Figure 2). The results show that with 100 and $50 \mu \mathrm{g} / \mathrm{mL}$ of Ini-HFBI aqueous solutions the reduction of interface tension proceeds in a similar way. However, at $10 \mu \mathrm{g} /$ $\mathrm{mL}$ concentration the reduction of surface tension was considerably slower, and the system was not stable even after $30 \mathrm{~min}$. Therefore, a concentration of $50 \mu \mathrm{g} / \mathrm{mL}$ was chosen for the experiments.

Compositional Characterization of the Surfaces. A simple method to verify surface modification is represented by the measurement of water contact angle (WCA) (Table S1). After silanization of a cleaned silicon wafer surface with chlorotrimethylsilane, WCA increased from $14.7^{\circ}$ to $95.6^{\circ}$, which indicates a change in the nature of the surface from hydrophilic to hydrophobic. The WCA then decreased to $35.6^{\circ}$ after deposition of Ini-HFBI layer, confirming that the protein adhered to the substrate through its hydrophobic patch and inverted its wettability. After growth of poly(PEGA) the WCA changed to $66.5^{\circ}$, which is in reasonable accord with values previously reported for surfaces modified with PEG-like acrylate polymers. ${ }^{47}$ Further information can be obtained by evaluating the surface free energy (SFE) and its polar $\left(\gamma^{\mathrm{P}}\right)$ and dispersive $\left(\gamma^{\mathrm{D}}\right)$ components through the Owens-Wendt method $^{44}$ (see Table S1 and the Supporting Information). As the silanol groups were reacted with chlorotrimethylsilane, a large decrease in polar SFE components was observed. The overall SFE and particularly its polar components then rose significantly as a result of the formation of an Ini-HFBI layer, confirming that the self-assembled film exposes the hydrophilic side of the protein which contains amino acids with polar and charged side chains.

Finally, the success of the in situ polymerization of poly(PEGA) is reflected by a further significant decrease in polar components since the polymer does not contain any charged moieties.

A more in-depth characterization of the chemical nature of the modified surfaces was obtained by XPS (Table 1 and

Table 1. Elemental Analysis As Derived from XPS for Different Functionalized Silicon Wafer Surfaces and the Relative Abundance of Different Components of the C 1s Peak (All Values in \%)

\begin{tabular}{|c|c|c|c|c|c|c|c|}
\hline \multirow[b]{2}{*}{ sample } & \multicolumn{4}{|c|}{ elemental analysis } & \multicolumn{3}{|c|}{ C 1s components } \\
\hline & C $1 \mathrm{~s}$ & O $1 \mathrm{~s}$ & Si $2 p$ & $\mathrm{~N} \mathrm{1s}$ & $\mathrm{C}-\mathrm{C}$ & $\mathrm{C}-\mathrm{O}$ & $\mathrm{C}=\mathrm{O}$ \\
\hline silanized silicon & 9.0 & 32.1 & 58.9 & & 86.0 & & \\
\hline $\begin{array}{l}\text { silanized silicon + } \\
\text { Ini-HFBI }\end{array}$ & 35.4 & 22.9 & 33.6 & 7.9 & 48.2 & 29.0 & 22.8 \\
\hline $\begin{array}{l}\text { silanized silicon }+ \\
\text { poly(PEGA)- } \\
\text { HFBI }\end{array}$ & 30.4 & 26.2 & 40.5 & 3.0 & 56.4 & 33.3 & 10.3 \\
\hline
\end{tabular}

Figure S3). The deposition of the protein layer over the silanized silicon is reflected by concurrent increase in carbon and decrease in $\mathrm{Si}$ content as well as by the appearance of nitrogen. This is accompanied by the onset of $\mathrm{C}-\mathrm{O}$ and $\mathrm{C}=\mathrm{O}$ components in the $\mathrm{C} 1 \mathrm{~s}$ peak, which is also consistent with the protein composition (Figure 3).

After polymerization there is a decrease of the amount of nitrogen, which is not present in poly(PEGA). $\mathrm{C}=\mathrm{O}$ functions are present in the polymer, but their relative amount is expected to be lower compared to the protein, as for poly (PEGA) the $\mathrm{C}=\mathrm{O} / \mathrm{C}-\mathrm{O}$ ratio is close to $1 / 16$. This is also confirmed by the analysis of the $\mathrm{C} 1 \mathrm{~s}$ peak components.

Finally, XPS data allow also some considerations about the thickness of the deposited layer. Because XPS typically probes a depth of ca. $10 \mathrm{~nm}$, the fact that a high amount of $\mathrm{Si}$ is still observed in the poly(PEGA)-functionalized surface clearly indicates that the average thickness of the layer is well below $10 \mathrm{~nm}$. This is further confirmed by the fact that the $\mathrm{C} / \mathrm{O}$ ratio is considerably smaller than the $2 / 1$ ratio, which is roughly expected for a pure poly(PEGA) surface, as well as from the AFM structural characterization, as described in the following paragraph.

Morphology of the Functionalized Surfaces. Poly(PEGA)-HFBI layers on silicon wafer surfaces were imaged by AFM to characterize their morphology and thickness. It has 


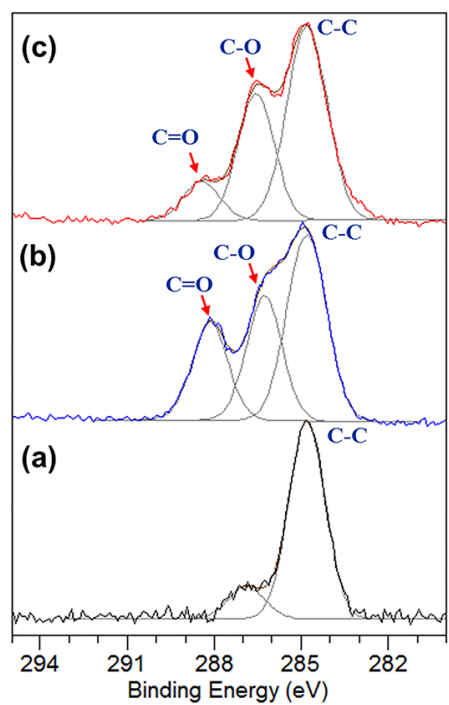

Figure 3. XPS C 1s spectra of (a) silanized Si surface, (b) Ini-HFBIcoated surface, and (c) poly(PEGA)-HFBI-coated surface.

been shown earlier that HFBI proteins form a full dense monolayer on hydrophobic surfaces. ${ }^{18,48}$ This ability is confirmed here for Ini-HFBI, as AFM images clearly show a difference between the smooth silanized silicon surface and the rougher surface evenly covered with Ini-HFBI (Figure 4a,b). The topography of the surface after in situ growth of the poly(PEGA) layer shows a different morphology with more extended and interconnected relief areas, which may suggest a tendency toward segregation of poly(PEGA) in the dry state (Figure 4c). The functionalization appeared to be quite homogeneous throughout the surfaces, as shown by the additional AFM and SEM images reported in Figures S4 and S5.

To estimate the thickness of the deposited layer, one sample was prepared by covering a portion of the silanized Si wafer with Teflon tape before functionalization with Ini-HFBI and poly(PEGA) and removing the tape delicately before AFM characterization in air. The image in Figure $4 \mathrm{~d}$ shows a clear edge between the two areas of the sample, and the section shows that the height of the combined protein and polymer layer is close to $2.5 \mathrm{~nm}$. This value is actually comparable to the thickness of the hydrophobin monolayer, ${ }^{49}$ which is in agreement with the considerations resulting from XPS and suggests that the poly(PEGA) chains collapse on the hydrophobin layer in the dry state, rather than forming a brush structure. This is not unexpected since there is only one polymerization initiator site on each hydrophobin molecule, and the section of a hydrophobin is roughly $2.2 \times 2.2 \mathrm{~nm}^{2}{ }^{9}{ }^{9,49}$ Therefore, the obtained polymer layer is predictably not dense enough to form a polymer brush. In spite of this relatively low density, the poly(PEGA) surface still displayed very good antifouling properties, as demonstrated by the protein adsorption studies below.

Antifouling Features and Reduction of Nonspecific Binding. The antifouling properties of the poly(PEGA)-HFBI surfaces were first studied by measuring the adsorption of BSA at $\mathrm{pH} 5.5$, which was reported to yield maximum adsorption on layers of the wild-type, nonconjugated protein HFBI. ${ }^{50}$ Compared to a surface only covered by HFBI, the adsorption of BSA was reduced almost completely in the presence of the poly(PEGA) layer (Figure 5), thus demonstrating excellent
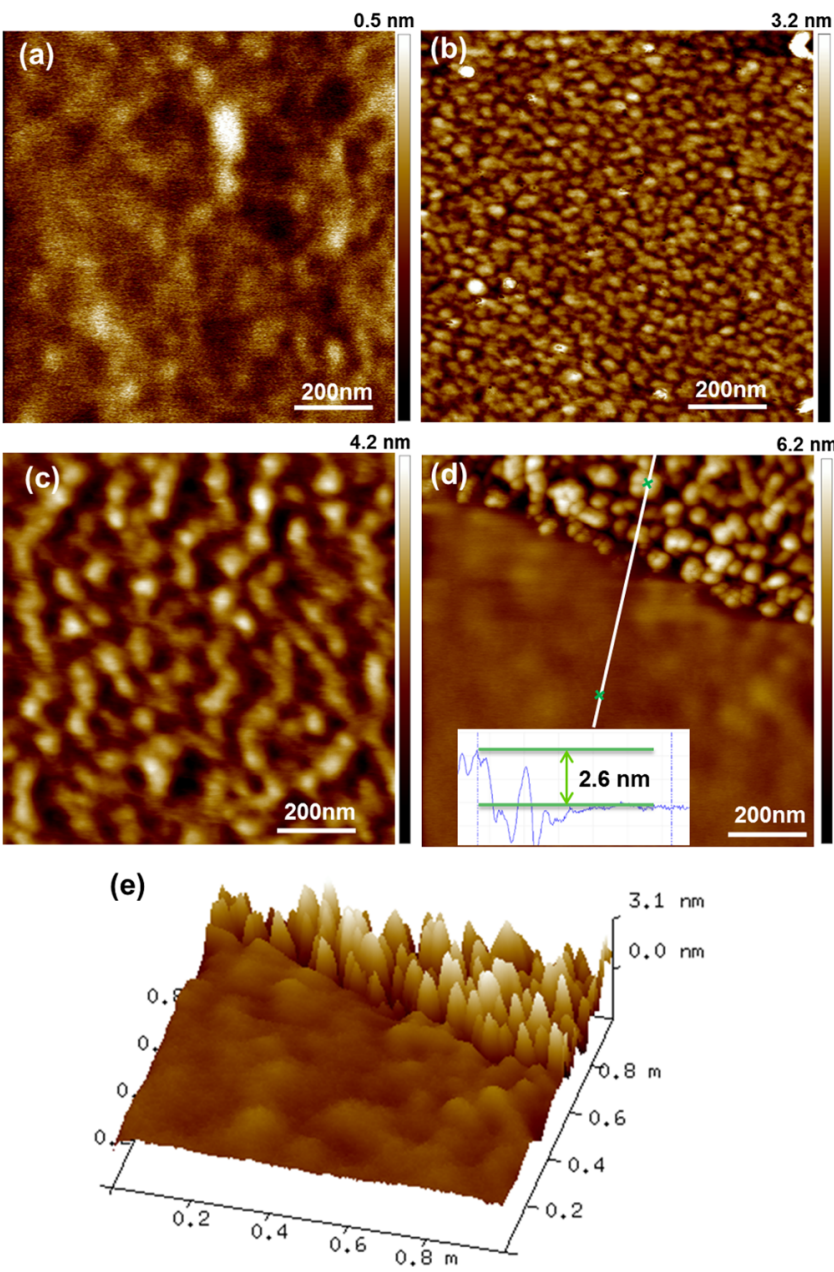

Figure 4. AFM images: (a) silanized Si wafer; (b) silanized Si wafer with an adsorbed layer of Ini-HFBI; (c) silanized Si wafer with a layer of poly(PEGA)-HFBI; (d) edge of the poly(PEGA)-HFBI layer (the silanized $\mathrm{Si}$ wafer was partially covered with Teflon tape before functionalization) and section along the line shown in panel d; (e) three-dimensional representation of the image in panel $\mathrm{d}$. All images show areas of $1 \times 1 \mu \mathrm{m}^{2}$.

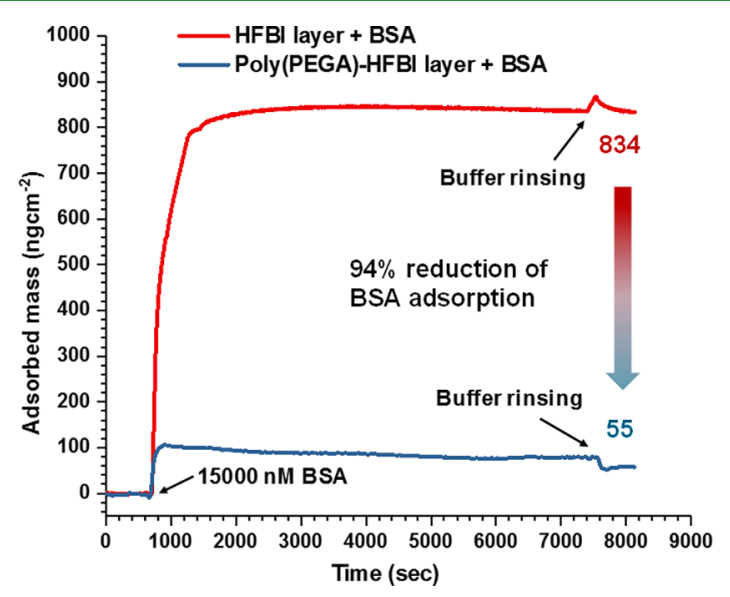

Figure 5. QCM-D measurements for antifouling performance: comparison of BSA adsorption on wild-type HFBI and poly(PEGA)-HFBI layers.

antifouling performance. The value indicated in Figure 5 for BSA adsorption on HFBI is significantly different from the one 
reported by Wang et al. (ca. $350 \mathrm{ng} \mathrm{cm}{ }^{-2}$ ). ${ }^{50}$ However, it should be noted that the two hydrophobin surfaces were prepared in very different ways. In Wang's case the assembly was performed directly inside the QCM instrument, by rapid fluxing of an HFBI solution followed by incubation for 30-60 min with ca. $40 \mu \mathrm{L}$ of the same solution (roughly corresponding to the volume of a QCM chamber); these conditions are ideal for the formation of a smooth HFBI layer. In our case a much larger volume of protein solution was used in the incubation step $(1 \mathrm{~mL})$, and the presence of an airwater interface may have caused the formation of some hydrophobin aggregates, which is a well-known phenomenon. ${ }^{51}$ These factors, together with possible shrinking effects upon drying of the deposited hydrophobin layer, ${ }^{43}$ may give rise to a considerably rougher surface, which may thus bind higher amounts of BSA.

Surfaces with antifouling features can be useful in biosensing applications. By saturating the nonspecific binding sites of a sensor surface with antifouling materials, it is possible to reduce the adsorption of nontarget compounds and thus increase detection specificity and sensitivity. ${ }^{52,53}$ In the area of biosensing, hydrophobins have been reported as an effective means for the immobilization of enzymes and antibodies. ${ }^{19-22,54,55}$ In particular, one fusion of HFBI with protein A was recently used in the preparation of highly sensitive sensing surfaces for IgG1 binding. ${ }^{23}$ Protein A is a highly stable surface receptor produced by Staphylococcus aureus, which is capable of binding the Fc portion of immunoglobulins with high affinity, especially IgGs, from a large number of species. ${ }^{56,57} \mathrm{We}$ investigated therefore the possibility to prepare IgG1-binding surfaces from mixed layers of this same fusion protein (HFBI-ProtA) with our poly(PEGA)HFBI bioconjugate and tested their ability to reduce nonspecific protein adsorption.

For a biosensor surface functionalized with a full monolayer of HFBI-ProtA, it is reasonable to assume that under conditions requiring high sensitivity measurement (i.e., low IgG1 concentrations and small sample volumes), not all available protein A units will actually bind IgG1. On the other hand, for significantly higher analyte concentrations the same will happen for sterical reasons due to the large size of IgG1 compared to HFBI-ProtA ( $150 \mathrm{kDa}$ vs $44 \mathrm{kDa})$. It follows that a portion of the HFBI-ProtA fusions can be replaced without significantly compromising the IgG1-binding capacity of the surface. To estimate the extent of the replaceable portion, we first studied by QCM-D the adsorption of mixed layers coassembled from solutions containing HFBI-ProtA and HFBI in different molar ratios, followed by the binding at $\mathrm{pH} 7$ of $\operatorname{IgG} 1$ injected in sequence at 8,80 , and $800 \mathrm{nM}$ concentration. Figure 6 shows the adsorbed mass increase at each step as well as the total adorbed mass, while the corresponding sensorgrams can be found in Figure S6. Based on the adsorbed mass of HFBI/HFBI-ProtA (blue blocks in Figure 6), a rough estimate of the actual amount of HFBI-ProtA adsorbed on the surface can be made. For molar ratios of $20 \%, 40 \%$, and $60 \%$ HFBI-ProtA in solution, the corresponding percentages in the adsorbed layer are estimated as $19 \%, 33 \%$, and $38 \%$ (see the Supporting Information and Table S2 for the details of the calculation). While these values should be considered as rough approximations, they appear realistic when considering that the larger size of protein A compared to HFBI will make it difficult for HFBI-ProtA alone to achieve a densely packed surface

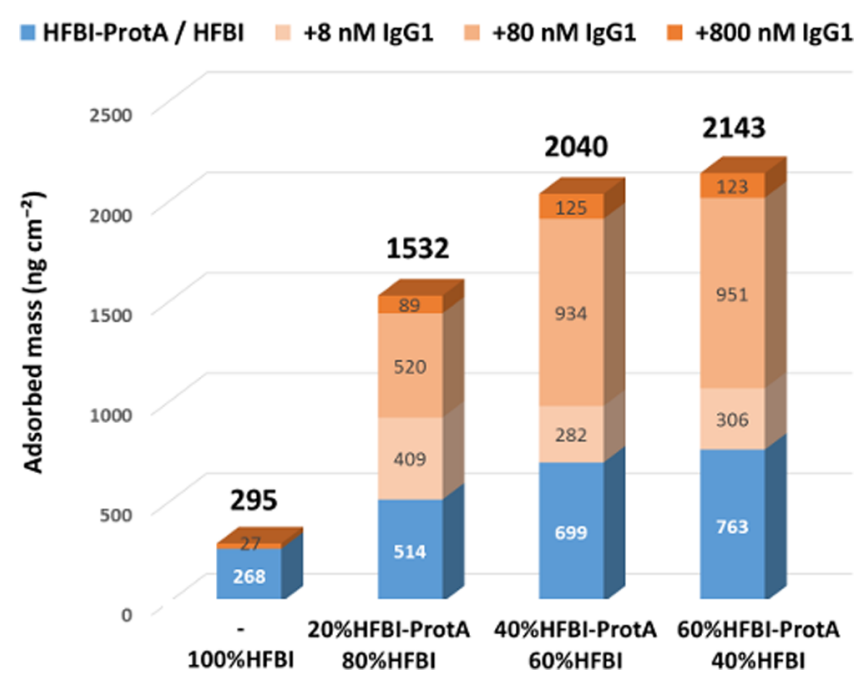

Figure 6. Average adsorbed mass as determined by QCM-D for hydrophobin layers coassembled from solutions having different HFBI-ProtA/HFBI molar ratios (blue) and subsequent binding of IgG1 injected in sequence at increasing concentration (shades of orange). The numbers inside the boxes indicate the increase in bound mass at each step, while the total adsorbed mass is shown above the columns.

layer, thus leaving empty spaces that can be filled by the smaller wild-type HFBI.

The mixed hydrophobin layers appeared quite homogeneous at the structural AFM analysis. No evident sign of segregation of HFBI-ProtA was observed, which would be expected to cause the appearance of higher patches due to the considerably larger size of the fusion protein ${ }^{23}$ (Figure S7); analogous observations can be made also for layers coassembled from IniHFBI and HFBI-ProtA (Figure S8). This homogeneity was further confirmed by incubating the coassembled surfaces with nanogold-labeled anti-IgG, which appeared to be welldistributed throughout the surfaces in the SEM images (Figure S9) and was present in larger quantity on surfaces prepared from solutions containing higher amounts of HFBI-ProtA.

The hydrophobin layers were exposed in sequence to increasing $\operatorname{IgG} 1$ concentrations (Figure 6 and Figure S6). Expectedly, very little IgG1 binding was observed when HFBIProtA was absent. The amount of IgG1 bound on the mixed layers increased with both immunoglobulin concentration and fraction of HFBI-ProtA in the layers. However, the adsorbed mass increased only slightly when the IgG1 concentration exceeded $80 \mathrm{nM}$ and for hydrophobin layers assembled from solutions containing more than $40 \%$ molar HFBI-ProtA (Figure 6 and Figure S6); this suggests that the maximum IgG1 binding capacity is already achieved at those values.

According to these observations, all following experiments were performed using protein mixtures containing $40 \%$ molar HFBI-ProtA. The structural homogeneity of these films was confirmed by SEM (Figure S10). At $\mathrm{pH} 5.5,373 \mathrm{ng} \mathrm{cm}^{-2}$ of BSA bound to a surface coassembled from an HFBI-ProtA/ HFBI mixture (Figure $7 \mathrm{a}$ and Figure S11) this value is lower than that observed for a pure HFBI surface (Figure 5), probably because HFBI-ProtA is somewhat less efficient than HFBI in binding BSA through electrostatic interaction at this $\mathrm{pH}$ (the pI's of HFBI and protein A are respectively $6.0^{58}$ and 4.8-5.0; that of BSA is 4.7). This still substantial adsorption is almost entirely prevented when poly(PEGA) is present. 
a

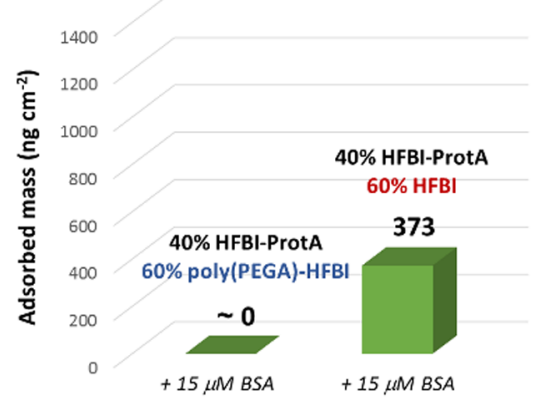

b

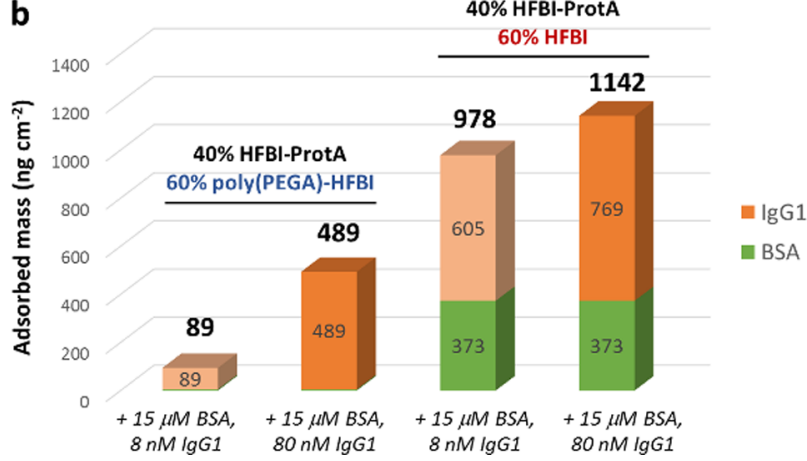

Figure 7. Average protein adsorption measured by QCM-D on mixed layers of HFBI-ProtA with either poly(PEGA)-HFBI or HFBI. The hydrophobin layers were initially coassembled from solutions containing 40\% molar HFBI-ProtA and 60\% molar of either Ini-HFBI or HFBI, respectively. (a) Adsorption of $15 \mu \mathrm{M}$ BSA; (b) sequential adsorption of $15 \mu \mathrm{M}$ BSA spiked with 8 and $80 \mathrm{~nm}$ IgG1: the total adsorbed mass is shown above the columns, while the estimated contributions from BSA and IgG1 are represented in green and shades of orange, respectively.

Therefore, a very good performance can still be obtained even when the surface is not fully covered by the poly(PEGA)-HFBI bioconjugate.

We then investigated the reduction of nonspecific adsorption by studying the competitive binding of BSA and IgG1 on the same surfaces. To do so, small amounts of immunoglobulin were added to the same BSA solution used

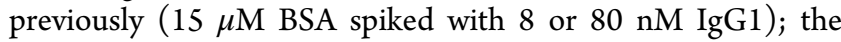
averaged results are shown in Figure $7 \mathrm{~b}$, while the corresponding sensorgrams are reported in Figure S12. By assuming that the adsorption of BSA is about the same as that shown in Figure $7 \mathrm{a}$, it follows that the adsorbed mass on the layer containing poly(PEGA)-HFBI is essentially due only to specific adsorption of IgG1, while in the absence of the antifouling polymer there is a substantial contribution from nonspecific BSA binding (shown in green in Figure $7 \mathrm{~b}$; the corresponding sensorgrams are reported in Figure S12). The presence of poly(PEGA) led to a partial reduction also of IgG1 binding under the conditions of our study, which was rather substantial at very low IgG1 concentration $(8 \mathrm{nM})$. However, this effect is already significantly reduced at $80 \mathrm{nM} \operatorname{IgG} 1$, and it should be remarked that in the absence of the antifouling polymer the false positive coming from nonspecific BSA adsorption is of comparable intensity to that of the signal due to specific IgG1 adsorption.

It should be remarked that antifouling performance measured against binary protein mixtures is not necessarily the same registered with more complex samples; ${ }^{59,60}$ some preliminary measurements suggested a lesser performance when animal serum was used (data not shown). A possible approach to improve this aspect is to increase the density of grafted polymer and will be the object of a future study. Nevertheless, the results reported here indicate that this strategy is promising to increase measurement sensitivity in biosensing devices. The use of poly(PEGA)-HFBI managed to prevent almost entirely the nonspecific adsorption of BSA and led to a remarkable enhancement of binding selectivity, making it possible to detect only the signal due to specific IgG1 binding even in samples where the immunoglobulin was a very minor component with respect to BSA.

\section{CONCLUSION}

In summary, the spontaneous self-assembly of hydrophobins was combined here with controlled radical polymerization concepts to prepare surfaces with antifouling and low nonspecific adsorption properties. The covalent, site-specific conjugation of a small-molecule polymerization initiator allowed the preparation of a hydrophobin-based macroinitiator, which retained the outstanding ability of the parent protein to self-assemble rapidly and spontaneously from aqueous solution on poorly reactive hydrophobic surfaces. The subsequent in situ polymerization of poly(PEGA) resulted in nanosized layers which displayed excellent antifouling performance, as demonstrated in QCM-D tests where BSA adsorption was reduced almost completely. These soft thin films show potential for use in biomaterial applications, e.g., for simple and fast deposition of nonfouling layers on PTFE, which is often used in biomaterials but has very poor reactivity. We further showed that by coassembling the same macroinitiator with a protein A-hydrophobin fusion and performing in situ polymerization of poly(PEGA), it is possible to prepare immunoglobulin-binding surfaces where the nonspecific adsorption of BSA from binary protein mixtures was essentially prevented. These surfaces greatly enhanced detection specificity for small volume samples featuring very low IgG1/BSA molar ratios. Even though also IgG1 binding was reduced to some extent on the surfaces containing the antifouling polymer, this effect can be expected to minimize through the optimization of e.g. the ratio between Ini-HFBI and HFBIProtA, the polymerization degree of poly(PEGA), and the experimental conditions in QCM-D binding experiments (e.g., sample flow rate and volume). These optimization aspects will be the object of ongoing and future studies.

The hydrophobin-polymer bioconjugate approach reported here shows promise for the preparation of biosensing devices with high specificity and sensitivity based on hydrophobin fusion proteins, which allow easy and rapid preparation of sensing layers, e.g., on the graphene electrodes of GFET devices $^{23}$ without requiring any surface activation procedure. Further investigations in this sense are ongoing in our laboratories.

\section{ASSOCIATED CONTENT}

\section{S Supporting Information}

The Supporting Information is available free of charge on the ACS Publications website at DOI: 10.1021/acsami.8b19968.

Cleaning and silanization procedure for silicon wafer, MALDI-TOF spectra of reduced NCysHFBI and IniHFBI, additional tensiometry measurements, table of water contact angle and surface free energy of cleaned 
and functionalized silicon wafer surfaces, XPS spectra of silanized $\mathrm{Si}$ surface before and after functionalization, additional AFM and SEM images of functionalized surfaces, QCM-D sensorgrams, calculations to estimate the percentages of different adsorbed hydrophobins in coassembled layers (PDF)

\section{AUTHOR INFORMATION}

\section{Corresponding Author}

*E-mail: roberto.milani@vtt.fi.

\section{ORCID}

Yingying Liu: 0000-0002-0013-9913

Kristian Kempe: 0000-0002-0136-9403

Paul Wilson: 0000-0002-9760-899X

Markus B. Linder: 0000-0002-7271-6441

David M. Haddleton: 0000-0002-4965-0827

Roberto Milani: 0000-0002-7997-7847

\section{Present Address}

K.K.: ARC Centre of Excellence in Convergent Bio-Nano Science and Technology, Monash Institute of Pharmaceutical Sciences, Monash University, VIC 3052, Parkville, Australia.

\section{Author Contributions}

Y.L. performed the experiments and wrote the manuscript with contributions from all authors. R.M. conceived the study, designed the experiments, and supervised the work. T.K.N., K.K., and P.W. contributed to experimental planning and interpretation of results. A.P. and L.S.J. contributed to characterization and interpretation of results. J.J.J., M.B.L., and D.M.H. contributed to work planning, interpretation of results, and overall supervision. All authors discussed, revised, and gave approval to the manuscript.

\section{Notes}

The authors declare no competing financial interest.

\section{ACKNOWLEDGMENTS}

This work was performed in collaboration with the Academy of Finland's Centres of Excellence Programme (2014-2019) and is supported by the Academy of Finland (project SelfSmart, decision no. 276537 and 284508). The authors thank gratefully Riitta Suihkonen (VTT) for hydrophobin production and purification, Dr. Katri Kurppa (VTT) for insightful discussions, and Dr. Nonappa (Aalto University) for the SEM images. K.K. gratefully acknowledges the award of a NHMRC-ARC Dementia Research Development Fellowship (APP1109945).

\section{REFERENCES}

(1) Hucknall, A.; Rangarajan, S.; Chilkoti, A. In Pursuit of Zero: Polymer Brushes That Resist the Adsorption of Proteins. Adv. Mater. 2009, 21 (23), 2441-2446.

(2) Jiang, S.; Cao, Z. Ultralow-Fouling, Functionalizable, and Hydrolyzable Zwitterionic Materials and Their Derivatives for Biological Applications. Adv. Mater. 2010, 22 (9), 920-932.

(3) Rodriguez-Emmenegger, C.; Houska, M.; Alles, A. B.; Brynda, E. Surfaces Resistant to Fouling from Biological Fluids: Towards Bioactive Surfaces for Real Applications. Macromol. Biosci. 2012, 12 (10), 1413-1422.

(4) Utrata-Wesolek, A. Antifouling Surfaces in Medical Application. Polimery 2013, 58 (9), 685-695.

(5) Groll, J.; Ademovic, Z.; Ameringer, T.; Klee, D.; Moeller, M. Comparison of Coatings from Reactive Star Shaped PEG-Stat-PPG Prepolymers and Grafted Linear PEG for Biological and Medical Applications. Biomacromolecules 2005, 6 (2), 956-962.
(6) Vaisocherová, H.; Brynda, E.; Homola, J. Functionalizable LowFouling Coatings for Label-Free Biosensing in Complex Biological Media: Advances and Applications. Anal. Bioanal. Chem. 2015, 407 (14), 3927-3953.

(7) Fenzl, C.; Genslein, C.; Domonkos, C.; Edwards, K. A.; Hirsch, T.; Baeumner, A. J. Investigating Non-Specific Binding to Chemically Engineered Sensor Surfaces Using Liposomes as Models. Analyst 2016, 141 (18), 5265-5273.

(8) Cui, M.; Wang, Y.; Jiao, M.; Jayachandran, S.; Wu, Y.; Fan, X.; Luo, X. Mixed Self-Assembled Aptamer and Newly Designed Zwitterionic Peptide as Antifouling Biosensing Interface for Electrochemical Detection of Alpha-Fetoprotein. ACS Sensors 2017, 2 (4), 490-494.

(9) Linder, M. B. Hydrophobins: Proteins That Self Assemble at Interfaces. Curr. Opin. Colloid Interface Sci. 2009, 14 (5), 356-363.

(10) Wösten, H. A. B.; Scholtmeijer, K. Applications of Hydrophobins: Current State and Perspectives. Appl. Microbiol. Biotechnol. 2015, 99 (4), 1587-1597.

(11) Khalesi, M.; Gebruers, K.; Derdelinckx, G. Recent Advances in Fungal Hydrophobin Towards Using in Industry. Protein J. 2015, 34 (4), 243-255.

(12) Szilvay, G. R.; Paananen, A.; Laurikainen, K.; Vuorimaa, E.; Lemmetyinen, H.; Peltonen, J.; Linder, M. B. Self-Assembled Hydrophobin Protein Films at the Air-Water Interface: Structural Analysis and Molecular Engineering. Biochemistry 2007, 46 (9), $2345-2354$

(13) Hakala, T. J.; Metsäjoki, J.; Granqvist, N.; Milani, R.; Szilvay, G. R.; Elomaa, O.; Deng, M.; Zhang, J.; Li, F. Adsorption and Lubricating Properties of HFBII Hydrophobins and Diblock Copolymer Poly(Methyl Methacrylate-b-Sodium Acrylate) Additives in Water-Lubricated Copper vs. a-C:H Contacts. Tribol. Int. 2015, 90, $60-66$.

(14) Askolin, S.; Linder, M.; Scholtmeijer, K.; Tenkanen, M.; Penttilä, M.; de Vocht, M. L.; Wösten, H. A. B. Interaction and Comparison of a Class I Hydrophobin from Schizophyllum Commune and Class II Hydrophobins Trichoderma Reesei. Biomacromolecules 2006, 7 (4), 1295-1301.

(15) Lumsdon, S. O.; Green, J.; Stieglitz, B. Adsorption of Hydrophobin Proteins at Hydrophobic and Hydrophilic Interfaces. Colloids Surf., B 2005, 44 (4), 172-178.

(16) Scholtmeijer, K.; Janssen, M. I.; van Leeuwen, M. B. M.; van Kooten, T. G.; Hektor, H.; Wösten, H. A. B. The Use of Hydrophobins to Functionalize Surfaces. Biomed. Mater. Eng. 2004, 14 (February), 447-454.

(17) Pigliacelli, C.; D’Elicio, A.; Milani, R.; Terraneo, G.; Resnati, G.; Baldelli Bombelli, F.; Metrangolo, P. Hydrophobin-Stabilized Dispersions of PVDF Nanoparticles in Water. J. Fluorine Chem. 2015, $177,62-69$.

(18) Gazzera, L.; Corti, C.; Pirrie, L.; Paananen, A.; Monfredini, A.; Cavallo, G.; Bettini, S.; Giancane, G.; Valli, L.; Linder, M. B.; Resnati, G.; Milani, R.; Metrangolo, P. Hydrophobin as a Nanolayer Primer That Enables the Fluorinated Coating of Poorly Reactive Polymer Surfaces. Adv. Mater. Interfaces 2015, 2 (14), 1500170.

(19) Corvis, Y.; Walcarius, A.; Rink, R.; Mrabet, N. T.; Rogalska, E. Preparing Catalytic Surfaces for Sensing Applications by Immobilizing Enzymes via Hydrophobin Layers. Anal. Chem. 2005, 77 (6), 16221630.

(20) Wang, Z.; Huang, Y.; Li, S.; Xu, H.; Linder, M. B.; Qiao, M. Hydrophilic Modification of Polystyrene with Hydrophobin for TimeResolved Immunofluorometric Assay. Biosens. Bioelectron. 2010, 26 (3), 1074-1079.

(21) Wang, X.; Wang, H.; Huang, Y.; Zhao, Z.; Qin, X.; Wang, Y.; Miao, Z.; Chen, Q.; Qiao, M. Noncovalently Functionalized MultiWall Carbon Nanotubes in Aqueous Solution Using the Hydrophobin HFBI and Their Electroanalytical Application. Biosens. Bioelectron. 2010, 26 (3), 1104-1108.

(22) Hennig, S.; Rödel, G.; Ostermann, K. Hydrophobin-Based Surface Engineering for Sensitive and Robust Quantification of Yeast Pheromones. Sensors 2016, 16 (5), 602. 
(23) Soikkeli, M.; Kurppa, K.; Kainlauri, M.; Arpiainen, S.; Paananen, A.; Gunnarsson, D.; Joensuu, J. J.; Laaksonen, P.; Prunnila, M.; Linder, M. B.; Ahopelto, J. Graphene Biosensor Programming with Genetically Engineered Fusion Protein Monolayers. ACS Appl. Mater. Interfaces 2016, 8 (12), 8257-8264.

(24) Piscitelli, A.; Cicatiello, P.; Gravagnuolo, A. M.; Sorrentino, I.; Pezzella, C.; Giardina, P. Applications of Functional Amyloids from Fungi: Surface Modification by Class I Hydrophobins. Biomolecules 2017, 7 (3), 45.

(25) Gravagnuolo, A. M.; Morales-Narváez, E.; Matos, C. R. S.; Longobardi, S.; Giardina, P.; Merkoçi, A. On-the-Spot Immobilization of Quantum Dots, Graphene Oxide, and Proteins via Hydrophobins. Adv. Funct. Mater. 2015, 25 (38), 6084-6092.

(26) Blättler, T. M.; Pasche, S.; Textor, M.; Griesser, H. J. High Salt Stability and Protein Resistance of Poly(L-Lysine)-g- Poly(Ethylene Glycol) Copolymers Covalently Immobilized via Aldehyde Plasma Polymer Interlayers on Inorganic and Polymeric Substrates. Langmuir 2006, 22 (13), 5760-5769.

(27) Li, L.; Chen, S.; Zheng, J.; Ratner, B. D.; Jiang, S. Protein Adsorption on Oligo(Ethylene Glycol)-Terminated Alkanethiolate Self-Assembled Monolayers: The Molecular Basis for Nonfouling Behavior. J. Phys. Chem. B 2005, 109 (7), 2934-2941.

(28) Harder, P.; Grunze, M.; Dahint, R.; Whitesides, G. M.; Laibinis, P. E. Molecular Conformation in Oligo(Ethylene Glycol)-Terminated Self-Assembled Monolayers on Gold and Silver Surfaces Determines Their Ability To Resist Protein Adsorption. J. Phys. Chem. B 1998, 102 (2), 426-436.

(29) Liu, L.; Li, W.; Liu, Q. Recent Development of Antifouling Polymers: Structure, Evaluation, and Biomedical Applications in Nano/Micro-Structures. Wiley Interdiscip. Rev. Nanomedicine Nanobiotechnology 2014, 6 (6), 599-614.

(30) Zhang, Q.; Wilson, P.; Li, Z.; Mchale, R.; Godfrey, J.; Waldron, C.; Haddleton, D. M. Aqueous Copper Mediated Living Polymerization: Exploiting Rapid Disproportionation of $\mathrm{CuBr}$ with $\mathrm{Me} 6$ TREN. J. Am. Chem. Soc. 2013, 135, 7355-7363.

(31) Deng, Y.; Li, Y.; Dai, J.; Lang, M.; Huang, X. Functionalization of Graphene Oxide towards Thermo-Sensitive Nanocomposites via Moderate in Situ SET-LRP. J. Polym. Sci., Part A: Polym. Chem. 2011, 49 (22), 4747-4755.

(32) Vorobii, M.; Pop-Georgievski, O.; de los Santos Pereira, A.; Kostina, N. Y.; Jezorek, R.; Sedláková, Z.; Percec, V.; RodriguezEmmenegger, C. Grafting of Functional Methacrylate Polymer Brushes by Photoinduced SET-LRP. Polym. Chem. 2016, 7 (45), 6934-6945.

(33) Jones, M. W.; Gibson, M. I.; Mantovani, G.; Haddleton, D. M. Tunable Thermo-Responsive Polymer-protein Conjugates via a Combination of Nucleophilic Thiol-ene "Click" and SET-LRP. Polym. Chem. 2011, 2 (3), 572-574.

(34) Zhang, Q.; Li, M.; Zhu, C.; Nurumbetov, G.; Li, Z.; Wilson, P.; Kempe, K.; Haddleton, D. M. Well-Defined Protein/Peptide-Polymer Conjugates by Aqueous Cu-LRP: Synthesis and Controlled SelfAssembly. J. Am. Chem. Soc. 2015, 137 (29), 9344-9353.

(35) Anastasaki, A.; Nikolaou, V.; Nurumbetov, G.; Wilson, P.; Kempe, K.; Quinn, J. F.; Davis, T. P.; Whittaker, M. R.; Haddleton, D. M. $\mathrm{Cu}(0)$-Mediated Living Radical Polymerization: A Versatile Tool for Materials Synthesis. Chem. Rev. 2016, 116 (3), 835-877.

(36) Shi, X.; Chen, G.; Wang, Y.; Yuan, L.; Zhang, Q.; Haddleton, D. M.; Chen, H. Control the Wettability of Poly (N Isopropylacrylamide- Co -1- Adamantan-1-Ylmethyl Acrylate) Modified Surfaces: The More Ada, the Bigger Impact? Langmuir 2013, 29, 14188-14195.

(37) Thomson, D. A. C.; Tee, E. H. L.; Tran, N. T. D.; Monteiro, M. J.; Cooper, M. A. Oligonucleotide and Polymer Functionalized Nanoparticles for Amplification-Free Detection of DNA. Biomacromolecules 2012, 13 (6), 1981-1989.

(38) Shi, Y.; Menzies, D. J.; Tsang, K. M.; Del Borgo, M. P.; Easton, C. D.; Aguilar, M. I.; Perlmutter, P.; Truong, V. X.; Forsythe, J. S. A Versatile and Rapid Coating Method via a Combination of Plasma Polymerization and Surface-Initiated SET-LRP for the Fabrication of
Low-Fouling Surfaces. J. Polym. Sci., Part A: Polym. Chem. 2017, 55 (15), 2527-2536.

(39) Szilvay, G. R.; Kisko, K.; Serimaa, R.; Linder, M. B. The Relation between Solution Association and Surface Activity of the Hydrophobin HFBI from Trichoderma Reesei. FEBS Lett. 2007, 581 (14), 2721-2726.

(40) Joensuu, J. J.; Conley, A. J.; Lienemann, M.; Brandle, J. E.; Linder, M. B.; Menassa, R. Hydrophobin Fusions for High-Level Transient Protein Expression and Purification in Nicotiana Benthamiana. Plant Physiol. 2010, 152 (2), 622-633.

(41) Weber, R. W.; Boutin, R. H.; Nedelman, M. A.; Lister-James, J.; Dean, R. T. Enhanced Kidney Clearance with an Ester-Linked 99mTc-Radiolabeled Antibody Fab'-Chelator Conjugate. Bioconjugate Chem. 1990, 1 (6), 431-437.

(42) Heredia, K. L.; Bontempo, D.; Ly, T.; Byers, J. T.; Halstenberg, S.; Maynard, H. D. In Situ Preparation of Protein-“Smart" Polymer Conjugates with Retention of Bioactivity. J. Am. Chem. Soc. 2005, 127, 16955-16960.

(43) Milani, R.; Pirrie, L.; Gazzera, L.; Paananen, A.; Baldrighi, M.; Monogioudi, E.; Cavallo, G.; Linder, M.; Resnati, G.; Metrangolo, P. A Synthetically Modified Hydrophobin Showing Enhanced Fluorous Affinity. J. Colloid Interface Sci. 2015, 448, 140-147.

(44) Owens, D. K.; Wendt, R. C. Estimation of the Surface Free Energy of Polymers. J. Appl. Polym. Sci. 1969, 13 (8), 1741-1747.

(45) Johansson, L. S.; Campbell, J. M. Reproducible XPS on Biopolymers: Cellulose Studies. Surf. Interface Anal. 2004, 36 (8), $1018-1022$.

(46) Beamson, G B. D. High Resolution XPS of Organic Polymers; Wiley: Chichester, 1992.

(47) Li, J.; Tan, D.; Zhang, X.; Tan, H.; Ding, M.; Wan, C.; Fu, Q. Preparation and Characterization of Nonfouling Polymer Brushes on Poly(Ethylene Terephthalate) Film Surfaces. Colloids Surf., B 2010, 78 (2), 343-350.

(48) De Stefano, L.; Rea, I.; Rendina, I.; Giocondo, M.; Houmadi, S.; Longobardi, S.; Giardina, P. Organic-Inorganic Interfaces for a New Generation of Hybrid Biosensors. Biosens. - Emerg. Mater. Appl. 2011, 311-332.

(49) Grunér, M. S.; Szilvay, G. R.; Berglin, M.; Lienemann, M.; Laaksonen, P.; Linder, M. B. Self-Assembly of Class II Hydrophobins on Polar Surfaces. Langmuir 2012, 28 (9), 4293-4300.

(50) Wang, Z.; Lienemann, M.; Qiau, M.; Linder, M. B. Mechanisms of Protein Adhesion on Surface Films of Hydrophobin. Langmuir 2010, 26 (11), 8491-8496.

(51) Gazzera, L.; Milani, R.; Pirrie, L.; Schmutz, M.; Blanck, C.; Resnati, G.; Metrangolo, P.; Krafft, M. P. Design of Highly Stable Echogenic Microbubbles through Controlled Assembly of Their Hydrophobin Shell. Angew. Chem., Int. Ed. 2016, 55 (35), 1026310267.

(52) Filipiak, M. S.; Rother, M.; Andoy, N. M.; Knudsen, A. C.; Grimm, S.; Bachran, C.; Swee, L. K.; Zaumseil, J.; Tarasov, A. Highly Sensitive, Selective and Label-Free Protein Detection in Physiological Solutions Using Carbon Nanotube Transistors with Nanobody Receptors. Sens. Actuators, B 2018, 255, 1507-1516.

(53) Gutiérrez-Sanz, Ó.; Andoy, N. M.; Filipiak, M. S.; Haustein, N.; Tarasov, A. Direct, Label-Free, and Rapid Transistor-Based Immunodetection in Whole Serum. ACS Sensors 2017, 2 (9), $1278-1286$.

(54) Piscitelli, A.; Pennacchio, A.; Cicatiello, P.; Politi, J.; De Stefano, L.; Giardina, P. Rapid and Ultrasensitive Detection of Active Thrombin Based on the Vmh2 Hydrophobin Fused to a Green Fluorescent Protein. Biosens. Bioelectron. 2017, 87, 816-822.

(55) Piscitelli, A.; Pennacchio, A.; Longobardi, S.; Velotta, R.; Giardina, P. Vmh2 Hydrophobin as a Tool for the Development of "Self-Immobilizing" Enzymes for Biosensing. Biotechnol. Bioeng. 2017, 114 (1), 46-52.

(56) Miyao, H.; Ikeda, Y.; Shiraishi, A.; Kawakami, Y.; Sueda, S. Immobilization of Immunoglobulin-G-Binding Domain of Protein A on a Gold Surface Modified with Biotin Ligase. Anal. Biochem. 2015, $484,113-121$ 
(57) Chiriacò, M. Serena; Primiceri, E.; De Feo, F.; Montanaro, A.; Monteduro, A. G.; Tinelli, A.; Megha, M.; Carati, D.; Maruccio, G. Simultaneous Detection of Multiple Lower Genital Tract Pathogens by an Impedimetric Immunochip. Biosens. Bioelectron. 2016, 79, 9-14.

(58) Milani, R.; Monogioudi, E.; Baldrighi, M.; Cavallo, G.; Arima, V.; Marra, L.; Zizzari, A.; Rinaldi, R.; Linder, M.; Resnati, G.; Metrangolo, P. Hydrophobin: Fluorosurfactant-like Properties without Fluorine. Soft Matter 2013, 9 (28), 6505.

(59) Riedel, T.; Riedelova-Reicheltova, Z.; Majek, P.; RodriguezEmmenegger, C.; Houska, M.; Dyr, J. E.; Brynda, E. Complete Identification of Proteins Responsible for Human Blood Plasma Fouling on Poly(Ethylene Glycol)-Based Surfaces. Langmuir 2013, 29 (10), 3388-3397.

(60) Rodriguez-Emmenegger, C.; Brynda, E.; Riedel, T.; Houska, M.; Subr, V.; Alles, A. B.; Hasan, E.; Gautrot, J. E.; Huck, W. T. S. Polymer Brushes Showing Non-Fouling in Blood Plasma Challenge the Currently Accepted Design of Protein Resistant Surfaces. Macromol. Rapid Commun. 2011, 32 (13), 952-957. 